\title{
Asymptotic Throughput in Wireless Multicast OFDM Systems
}

\author{
Juan Liu*, Wei Chen ${ }^{\dagger}$, Member, IEEE, Zhigang $\mathrm{Cao}^{\dagger}$, Senior Member, IEEE, \\ Ying Jun (Angela) Zhang ${ }^{\ddagger}$, Member, IEEE, Soung Chang Liew ${ }^{\ddagger}$, Senior Member, IEEE \\ Email:*liujuan05@mails.tsinghua.edu.cn $\dagger\{$ wchen, czg-dee $\} @$ tsinghua.edu.cn \\ $\ddagger\{$ yjzhang, soung $\} @$ ie.cuhk.edu.hk
}

\begin{abstract}
With the proliferation of wireless multimedia applications, multicast/broadcast has been recognized as an efficient technique to transmit a large volume of data to multiple mobile stations at the same time. In most multicast systems, the transmitter (e.g., base station) adapts its data rate to the worst channel among all users in the multicast group, so as to guarantee service quality to each user. Predictably, the more users in a multicast group, the lower data rate the base station can transmit. On the other hand, grouping more users together leads to a more efficient utilization of spectrum bandwidth, as these users are served simultaneously. A natural question that arises is how to group users to maximize the throughput of multicast systems, given a fixed amount of bandwidth resource. In this paper, we attempt to answer this important question that has not been addressed before. Through theoretical analysis, we prove that (1) the average throughput increases with the number of users in a multicast group, when the number of subcarriers allocated to a group is proportional to the number of users therein. Moreover, the throughput approaches infinite-bandwidth Gaussian channel capacity when the number of users gets large; (2) the number of users, and hence the number of subcarriers, that is needed for throughput to be arbitrarily close to its asymptotic value increases almost linearly with the transmit SNR. Our analysis is validated through simulations.
\end{abstract}

\section{INTRODUCTION}

The explosive growth of wireless broadband multimedia services has placed an unprecedented demand on the already scarce wireless spectrum bandwidth [1]. Hence, efficient utilization of the scarce spectrum resource has become a key challenge in the design of next-generation wireless systems. It is worth noting that many emerging applications such as mobile TV and group oriented mobile commerce aim to deliver the same large volume of data to multiple users in the network [2]. Such applications are particularly well suited for multicast transmission, which broadcasts the same content to a group of users at a single transmission. Conceivably, point-to-multipoint multicast transmission can greatly improve the spectrum efficiency compared to the point-to-point unicast transmission. As a matter of fact, multicast/broadcast features have been included in the recent release of IEEE 802.16e WiMax standard [3], which adopts Orthogonal Frequency Division Multiplexing (OFDM) as the air interface to combat multi-path fading effect [4].

In wireless networks, multicast transmission to a group of users benefits from the broadcast nature of radio channels. The

This work is supported in part by NSFC/RGC joint funding under Grants No. 60618001. achievable rate region of these users was derived for many classes of broadcast channels through maximin, time-sharing and superposition procedures in [5]. In order to maximize users' aggregate throughput in fading broadcast channels, [6] and [7] investigated the capacity region and the optimal power allocation scheme that can be achieved by superposition coding as well as successive decoding. The above work mainly aimed at enlarging the achievable rate region when one group of users shared the broadcast channels. Focusing on the situation when multiple groups compete for one broadcast channel, Sharif et al proposed three scheduling schemes to maximize the system throughput while maintaining the rational rate constraints on these groups, wherein all users have the same data rate [8]. More recently, for cellular data networks with multiple Time Division Multiplexing (TDM) channels, two proportional fair multicast scheduling algorithms were proposed by Won et al to select an appropriate multicast data rate and a group for transmission in each slot [9]. When the algorithms in [8], [9] are applied, correct reception of multicast packets cannot be guaranteed for the users suffering from severe channel fading in each multicast group.

In this paper, we consider multicast in access networks where a base station (BS) or access point (AP) broadcasts data to a group of users, referred to as a multicast group. To ensure the signal reception quality at each user, one method is to adapt the data transmission rate to the worst channel among all users in the multicast group. Predictably, the data transmission rate decreases with the number of users in a group. On the other hand, in a system with fixed number of channels (e.g., subcarrier in OFDM systems) and fixed user population, the bandwidth resource allocated to a group is proportional to the number of users in the group. With the two factors counteracting each other, it is important to understand the following question: how to form multicast groups and how large should a multicast group be to maximize the average throughput that each user can receive. The answer to this question would serve as a guideline to scheduling transmissions in wireless multicast systems. To the best of our knowledge, such problem has not be investigated before.

Assuming that each user perceives an i.i.d. Rayleigh fading channel, we reach the following key results in this paper. First, the average throughput received by each user increases with the number of users in a multicast group. In other words, we should group as many users as possible whenever they request the same data from the BS. The throughput 


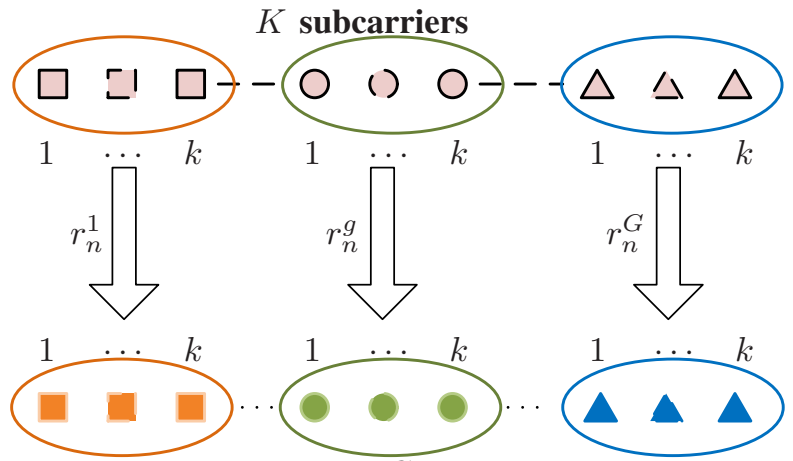

$K$ users divided into $G$ multicast groups

Fig. 1. Every multicast group with $k$ users are allocated $k$ fixed subcarriers in a wireless OFDM system. The BS transmits data to the users on subcarrier $n$ at a transmission rate $r_{n}^{g}$ for the $g$-th multicast group.

asymptotically approaches a finite value, which is equal to the capacity of infinite-bandwidth AWGN channel, when the number of users in a group becomes large. Through rigorous analysis, we find that the number of users that is needed for the throughput to be arbitrarily close to its asymptotic value increases almost linearly with the average transmit Signal-toNoise Ratio (SNR). The analysis is shown to be quite accurate through numerical simulations.

The rest of this paper is organized as follows. Section II introduces the system model. In Section III, we analyze the average throughput as a function of the size of the multicast group. The number of users needed to achieve the asymptotic throughput is analyzed in Section IV. In the end, the paper is concluded in Section V.

\section{SySTEM Model}

Consider a wireless OFDM multicast system with $J$ subcarriers and $K$ users requiring the same desirable program from the BS. The users are equally divided into $G$ multicast groups. Assuming that $K$ is divisible by $G$ and $J$ is an integer multiple of $K$, each multicast group is associated with $k=K / G$ users and $j k$ subcarriers, where $j=J / K$. For simplification, we assume $J=K$ in the rest of the paper, as shown in Fig. 1. Our results, however, easily extends to the case with $j>1$. We further assume that equal power is transmitted on all subcarriers.

Let $S C_{m, n}^{g}(g=1, \cdots, G ; m=1, \cdots, k ; n=1, \cdots, k)$ denote the sub-channel of user $m$ of the $g$-th multicast group on subcarrier $n$ with a stationary and ergodic time-varying power gain $\alpha_{m, n}^{g}$. The power gains $\alpha_{m, n}^{g}$ for all the $m$ and $n$ are assumed to follow an i.i.d. standard exponential distribution (Rayleigh fading). It is assumed that a perfect instantaneous channel estimation is made in every user's receiver and is transmitted back to the BS through errorfree feedback path. So the channel gain $\alpha_{m, n}^{g}$ is known both to the BS and the receiver. Let $P$ denote the average transmit signal power on each subcarrier, $N_{0}$ denote the singlesided power spectral density of Additive White Gaussian Noise (AWGN), and $B$ denote the bandwidth of each subchannel. Let $\gamma_{t}=P /\left(N_{0} B\right)$ denote the average transmitterside SNR. Then the instantaneous received SNR of $S C_{m, n}^{g}$ is represented by $\gamma_{m, n}^{g}=\alpha_{m, n}^{g} P /\left(N_{0} B\right)=\alpha_{m, n}^{g} \gamma_{t}$. Based on Shannon's channel capacity, the instantaneous capacity of each sub-channel $S C_{m, n}^{g}$ can be represented by $c_{m, n}^{g}=$ $B \log _{2}\left(1+\gamma_{m, n}^{g}\right)=B \log _{2}\left(1+\alpha_{m, n}^{g} \gamma_{t}\right)$.

As mentioned earlier, the data rate at which the BS transmits to a multicast group is determined by the worst channel in the group. In other words, the data rate transmitted to group $g$ on subcarrier $n$, denoted as $r_{n}^{g}$, is given by $r_{n}^{g}=\min _{m} c_{m, n}^{g}$. To be more precise, $r_{n}^{g}=B \log _{2}\left(1+\gamma_{n}^{g}\right)$, where $\gamma_{n}^{g}=\alpha_{n}^{g} \gamma_{t}$ and $\alpha_{n}^{g}=\min _{m} \alpha_{m, n}^{g}$. As each group occupies $k$ subcarriers, the total instantaneous data rate transmitted to each user is given by $R_{k}^{g}=\sum_{n=1}^{k} B \log _{2}\left(1+\alpha_{n}^{g} \gamma_{t}\right)$. As the users in the same multicast group enjoy the same throughput, the aggregate instantaneous throughput for the $g$-th multicast group is exactly $k R_{k}^{g}$. Thus, the $K$ users' total throughput can be represented by $C_{\text {total }}=\sum_{g=1}^{G} k R_{k}^{g}$. Clearly, $C_{\text {total }}$ is a random variable as it is a function of random variables $\alpha_{n}^{g}(\forall g, n)$. In the next section, we are going to investigate the expected throughput that is received by one user as a function of $k$.

\section{Average Throughput of One User}

Assuming each user perceives i.i.d. Rayleigh fading channels, the average throughput of one user is given by (1), where the expectation is taken over channel fading.

$$
\begin{aligned}
C & =\mathbf{E}\left(\sum_{g=1}^{G} k R_{k}^{g} / K\right) \\
& =\mathbf{E}\left(\sum_{g=1}^{G} k \sum_{n=1}^{k} B \log _{2}\left(1+\alpha_{n}^{g} \gamma_{t}\right) / K\right) \\
& \stackrel{1 a}{=} k \mathbf{E}\left(B \log _{2}\left(1+\alpha \gamma_{t}\right)\right) \\
& =k \int_{\alpha} B \log _{2}\left(1+\gamma_{t} \alpha\right) p(\alpha) d \alpha
\end{aligned}
$$

where $1 a$ follows from substituting all the i.i.d. $\alpha_{n}^{g}$ with the variable $\alpha$ that has the probability distribution $p(\alpha)$.

As mentioned above, $\alpha$ is the smallest order statistics of $k$ i.i.d. random variables $X_{1}, X_{2}, \cdots, X_{k}$ following the standard exponential distribution, each with probability density function (pdf) $f(x)$ and cumulative distribution function (cdf) $F(x)$. From [10], the cdf $F_{\alpha}(x)$ of $\alpha$ is given by

$$
\begin{aligned}
F_{\alpha}(x) & =\operatorname{Pr}(\alpha \leq x)=1-\operatorname{Pr}(\alpha>x) \\
& =1-\operatorname{Pr}\left(\text { all } X_{i}>x\right)=1-[1-F(x)]^{k} .
\end{aligned}
$$

Then, the probability distribution of the variable $\alpha$ follows that

$$
p_{\alpha}(x)=\partial F_{\alpha}(x) / \partial x=k[1-F(x)]^{k-1} f(x) .
$$

Substituting $X_{i}$ 's $f(x)$ and $F(x)$ into (3), $p_{\alpha}(x)$ is given by

$$
p_{\alpha}(x)= \begin{cases}k \exp (-k x), & \text { if } x \geq 0 \\ 0, & \text { if } x<0 .\end{cases}
$$

Substituting (4) into (1), we have

$$
\begin{aligned}
C & =\frac{B k^{2}}{\ln 2} \int_{0}^{\infty} \ln \left(1+\gamma_{t} x\right) e^{-k x} d x \\
& \stackrel{5 a}{=} \frac{B k^{2}}{\gamma_{t} \ln 2} \int_{1}^{\infty} e^{-k \frac{y-1}{\gamma_{t}}} \ln y d y \\
& \stackrel{5 b}{=} \frac{B \gamma_{t}}{\ln 2} a e^{a}\left(\int_{a}^{\infty} e^{-z} \ln z d z-e^{-a} \ln a\right)
\end{aligned}
$$


where $5 a$ follows from $y=1+\gamma_{t} x$ and $5 b$ follows from $z=k y / \gamma_{t}$ and $a=k / \gamma_{t}$. Given $\gamma_{t}$ and $B$, the user's average throughput $C$ is re-written as a function of the size of a multicast group, $k$,

$$
C(k)=\frac{B k}{\ln 2} e^{k / \gamma_{t}}\left(\int_{k / \gamma_{t}}^{\infty} e^{-z} \ln z d z-e^{-k / \gamma_{t}} \ln \frac{k}{\gamma_{t}}\right) .
$$

Though the integral of the product of an exponential function and a logarithmic one in (6) is very difficult to figure out, the change of $C(k)$ with $k$ can be further investigated as follows.

In Theorem 1, we prove that $C(k)$ is a monotonically increasing function of the number of users (or the number of subcarriers) $k$ in a multicast group. We first present in Lemma 1 an inequality that is needed in the proof of Theorem 1.

Lemma 1: For any $a>0$, the following inequality holds.

$$
(1+a) e^{a} \int_{a}^{\infty} \frac{e^{-z}}{z} d z>1 .
$$

Proof: From $(1+z)^{2}>z^{2}+2 z$, we have

$$
\frac{1}{z}>\frac{1}{(1+z)^{2}}+\frac{1}{1+z}
$$

for any $z \geq a>0$. Multiplying by $e^{-z}$ and then integrating over $z$ on both sides of (8), we have

$$
\int_{a}^{\infty} \frac{e^{-z}}{z} d z>\int_{a}^{\infty}\left(\frac{e^{-z}}{(1+z)^{2}}+\frac{e^{-z}}{1+z}\right) d z=(1+a)^{-1} e^{-a} .
$$

Therefore, the inequality (7) holds for any $a>0$.

Theorem 1: Given $B$ and $\gamma_{t}, C(k)$ is a monotonically increasing function of $k$.

Proof: A function is monotonic increasing if its first derivative is non-negative. From (5), we have

$$
\begin{aligned}
& C^{\prime}(k)=\frac{\partial C(k)}{\partial a} \frac{\partial a}{\partial k} \\
& =\frac{B}{\ln 2}\left[(1+a) e^{a} \int_{a}^{\infty} e^{-z} \ln z d z-(1+a) \ln a-1\right] \\
& =\frac{B}{\ln 2}\left[(1+a) e^{a} \int_{a}^{\infty} \frac{e^{-z}}{z} d z-1\right]
\end{aligned}
$$

where $a=k / \gamma_{t}$. In addition, from the inequality (7), $C^{\prime}(k)>$ 0 always holds for any $k>0$. So the average capacity $C(k)$ is a monotonically increasing function as $k$ increases.

As $C(k)$ increases with $k$, each user can enjoy a higher throughput when users are divided into larger multicast groups. In this regard, the best grouping strategy is to put all users requiring the same program into one multicast group. In the next section, we will investigate the asymptotic behavior of $C(k)$ when $k$ becomes large.

\section{Performance Analysis}

\section{A. Asymptotic Throughput}

In Theorem 2, we compute the asymptotic value of $C(k)$ as $k$ approaches infinity.

Theorem 2: As $k \rightarrow \infty, C(k) \rightarrow C_{\infty}$, where $C_{\infty}$ equals to the capacity of the infinite-bandwidth AWGN channel with the same average transmitted power $P$.
Proof: From Theorem 1, $C\left(k_{1}\right) \leq C\left(k_{2}\right)$ for any $0<$ $k_{1} \leq k_{2}$. Denote $C_{\infty}=\lim _{k \rightarrow \infty} C(k)$, which is thus an upper bound of $C(k)$ for any given $k>0$. A detailed computation of $C_{\infty}$ is carried out as follows

$$
\begin{aligned}
& C_{\infty}=\lim _{k \rightarrow \infty} C(k) \\
& \stackrel{10 a}{=} \lim _{k \rightarrow \infty} \frac{B}{\ln 2} \frac{\left(\int_{k / \gamma_{t}}^{\infty} e^{-z} \ln z d z-\ln \frac{k}{\gamma_{t}} e^{-k / \gamma_{t}}\right)^{\prime}}{\left(k^{-1} e^{-k / \gamma_{t}}\right)^{\prime}} \\
& =\lim _{k \rightarrow \infty} \frac{B}{\ln 2\left(\frac{1}{k}+\frac{1}{\gamma_{t}}\right)}=\frac{1}{\ln 2} B \gamma_{t}=\frac{P}{N_{0} \ln 2} \text { bits/s }
\end{aligned}
$$

where we have used L'Hospital's rule in 10a. We see that the user's average throughput $C(k)$ is convergent to an asymptotic value $C_{\infty}$ when the number of subcarriers $k$ increases to infinity. Interestingly, we note that when the bandwidth $W$ of the AWGN channel approaches infinity, the channel capacity with the average transmitted power $P$ satisfies $\lim _{W \rightarrow \infty} W \log _{2}\left(1+\frac{P}{W N_{0}}\right)=\frac{P}{N_{0} \ln 2}$ bits/s [11]. Thus, $C_{\infty}$ is essentially equal to the capacity of the infinite-bandwidth AWGN channel.

From (10), we see that the user's average throughput $C(k)$ approaches the constant asymptotic throughput $C_{\infty}$ for a sufficiently large $k$. Thus, the expected throughput of each user cannot be arbitrarily increased even if it is associated with a multicast group with infinite users, and hence allocated infinite number of subcarriers. This is due to the fact that the data transmission rate on each subcarrier approaches zero asymptotically.

\section{B. $\epsilon$-Threshold Multicast Group Size}

In this subsection, we investigate how large the multicast group should be in order to have $C(k)$ close to its asymptotic value $C_{\infty}$. Substituting $a=k / \gamma_{t}$ into (9), it is easily seen that the first term in the bracket becomes

$\lim _{k \rightarrow \infty}\left(1+\frac{k}{\gamma_{t}}\right) e^{\frac{k}{\gamma_{t}}} \int_{\frac{k}{\gamma_{t}}}^{\infty} \frac{e^{-z}}{z} d z=\lim _{k \rightarrow \infty} \frac{\left(\int_{\frac{k}{\gamma_{t}}}^{\infty} \frac{e^{-z}}{z} d z\right)^{\prime}}{\left(\left(1+\frac{k}{\gamma_{t}}\right)^{-1} e^{-\frac{k}{\gamma_{t}}}\right)^{\prime}}=1$, where L'Hospital's rule has been applied. Thus, the first derivative of $C(k)$ approaches zero when $k$ becomes large. Thus, for any $\epsilon>0$, there must exist

$$
C^{\prime}(k) \leq \epsilon, \quad \forall k \in \mathbf{Z}^{+}, k \geq \delta .
$$

$C(k)$ is said to be close to its asymptotic value $C_{\infty}$ when $C^{\prime}(k)$ is close to zero. Note that $\delta$ is the multicast group size that is needed for $C^{\prime}(k)$ to be smaller than a small value $\epsilon$. Hence, we refer to $\delta$ as the $\epsilon$-threshold multicast group size. In Lemma 2 and Theorem 3, it is proved that $C^{\prime}(k)$ is a monotonically decreasing function of $k$.

Lemma 2: For any $a>0$, the following inequality holds.

$$
(2+a) e^{a} \int_{a}^{\infty} \frac{e^{-z}}{z} d z<1+\frac{1}{a} .
$$

Proof: For $z \geq a>0$, we have $z^{4}+4 z^{3}+4 z^{2}<z^{4}+$ $4 z^{3}+4 z^{2}+4 z$ and thus

$$
\frac{1}{z}<\frac{1}{2 z}+\frac{1}{2 z^{2}}+\frac{1}{2(z+2)}+\frac{1}{2(z+2)^{2}} .
$$




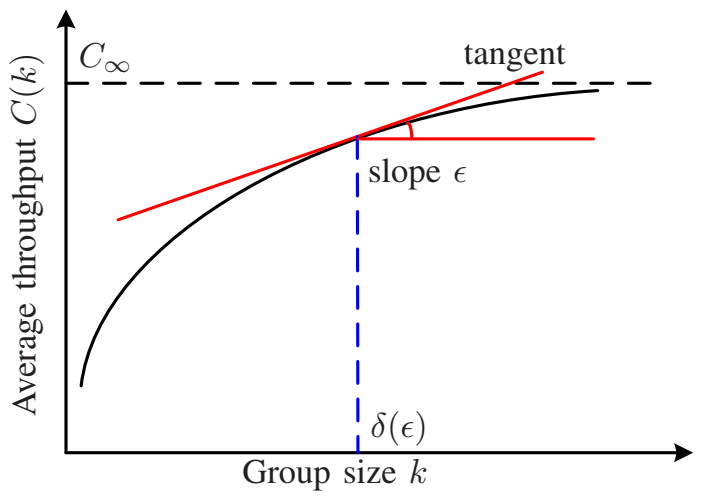

Fig. 2. The $\epsilon$-threshold multicast group size.

Multiplying by $e^{-z}$ and then integrating over $z$ on both sides of (13), we see that

$$
\int_{a}^{\infty} \frac{e^{-z}}{z} d z<\left(\frac{1}{2 a}+\frac{1}{2(a+2)}\right) e^{-a},
$$

and inequality (12) follows.

Theorem 3: Given $B$ and $\gamma_{t}$, the first derivative of $C(k)$ is a monotonically decreasing function of $k$.

Proof: By (9), we compute the second derivative of $C(k)$ as

$$
C^{\prime \prime}(k)=\frac{B}{\gamma_{t} \ln 2}\left[(2+a) e^{a} \int_{a}^{\infty} e^{-z} \ln z d z-\frac{1+a}{a}\right]
$$

From (12), $C^{\prime \prime}(k)<0$ holds for any $B$ and $\gamma_{t}$. Thereby, $C^{\prime}(k)$ is a monotonically decreasing function of $k$.

Though the monotonic function $C^{\prime}: \mathbf{Z}^{+} \rightarrow \mathbf{R}^{+}$is an injective mapping, by extending its field of definitions from integer to real, its inverse function $\left(C^{\prime}\right)^{-1}: \mathbf{R}^{+} \rightarrow \mathbf{R}^{+}$can be defined. Thus the integer group size $\delta$ can be written as a function of $\epsilon$

$$
\delta(\epsilon)=\left\lceil\left(C^{\prime}\right)^{-1}(\epsilon)\right\rceil, \epsilon>0
$$

where $\lceil\cdot\rceil$ is the ceiling function. Here, we ignore $\epsilon=$ $\lim _{k \rightarrow \infty}\left(C^{\prime}\right)(k)=0$ at infinite $k$. It shows in Fig. 2 that $\epsilon$ is the slope of the continuous $C(k)$ curve, and it can be regarded as a measure of the distance between the user's average throughput $C(\delta(\epsilon))$ and $C_{\infty} . C(\delta(\epsilon))$ is closer to $C_{\infty}$ for smaller $\epsilon$.

Next, the property of $\delta(\epsilon)$ is discussed in the following.

Theorem 4: For any $\epsilon>0$, the corresponding number of subcarriers $\delta(\epsilon)$ increases near-linearly with $\gamma_{t}$ for fixed bandwidth $B$.

Proof: Given $\epsilon>0$, a real value $k_{x}$ is computed by solving the equation $C^{\prime}\left(k_{x}\right)=\epsilon$ as follows. Substituting (9) into the equation yields

$$
\frac{B}{\ln 2}\left[\left(1+a_{x}\right) e^{a_{x}} \int_{a_{x}}^{\infty} \frac{e^{-z}}{z} d z-1\right]=\epsilon
$$

where $a_{x}=k_{x} / \gamma_{t}$. Then we have

$$
\int_{a_{x}}^{\infty} \frac{e^{-z}}{z} d z=\eta\left(1+a_{x}\right)^{-1} e^{-a_{x}}
$$

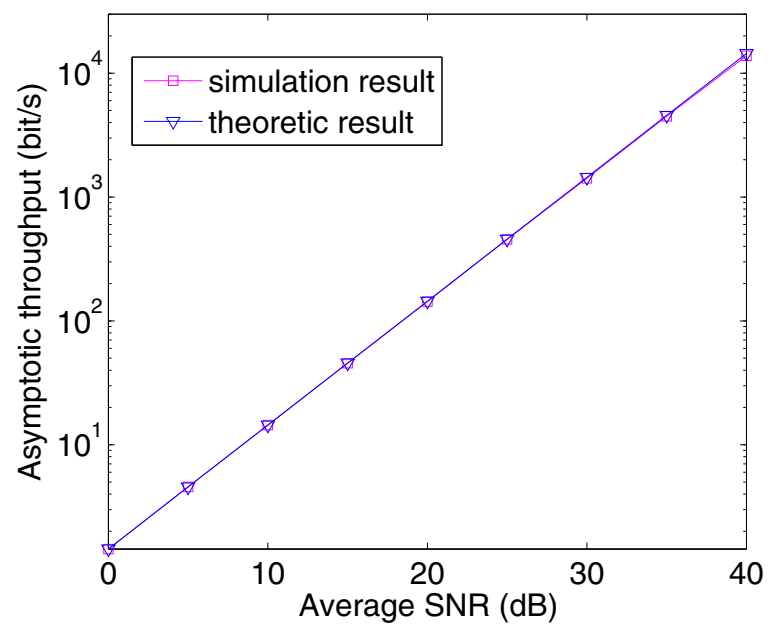

Fig. 3. The asymptotic throughput $C_{\infty}$.

where $\eta=1+\frac{\epsilon \ln 2}{B}$ is a constant for the given $\epsilon$. By differentiating the both sides of (17), we have

$$
-\frac{e^{-a_{x}}}{a_{x}}=-\eta\left(a_{x}+a_{x}\right)^{-2} e^{-a_{x}}-\eta\left(1+a_{x}\right)^{-1} e^{-a_{x}} .
$$

It follows that $\left(1+a_{x}\right)^{2}=\eta a_{x}+\eta a_{x}\left(1+a_{x}\right)$. Then we obtain $a_{x}=\sqrt{1+\frac{B}{\epsilon \ln 2}}-1$, and thus

$$
k_{x}=\left(\sqrt{1+\frac{B}{\epsilon \ln 2}}-1\right) \gamma_{t} .
$$

Therefore the real number $k_{x}$ increases linearly with the average SNR $\gamma_{t}$ for given $\epsilon$. From (15), $\delta(\epsilon)$ is given by

$$
\delta(\epsilon)=\left\lceil\left(\sqrt{1+\frac{B}{\epsilon \ln 2}}-1\right) \gamma_{t}\right\rceil
$$

From (18) and (19), it satisfies $0 \leq \delta(\epsilon)-k_{x}<1$. So for any $\epsilon>0, \delta(\epsilon)$ is a near-linear function of the average SNR $\gamma_{t}$.

It is obvious from (19) that for any $\epsilon>0, \delta(\epsilon)$ increases with $\gamma_{t}$. Moreover, the smaller $\epsilon$, the larger group size is needed for given $\gamma_{t}$. When $\epsilon$ is small enough, its reciprocal becomes so large that some items in (19) can be omitted and $\delta(\epsilon)$ can be re-written as

$$
\delta^{\prime}(\epsilon)=\left\lceil\frac{B^{1 / 2} \gamma_{t}}{(\ln 2)^{1 / 2}} \epsilon^{-1 / 2}\right\rceil .
$$

Thus, for any $B$ and $\gamma_{t}, \delta^{\prime}(\epsilon)$ decreases near-linearly with $\epsilon$ on a $\log -\log$ scale for $\epsilon$ comparatively small.

\section{Numerical Results}

In this section, the analytical results in Section III and IV are validated via simulations. Unless otherwise stated, each subcarrier's bandwidth $B$ is set to unity in the following simulations. In Fig. 3, the asymptotic throughput $C_{\infty}$ is plotted as a function of transmitter-side SNR $\gamma_{t}$. The figure confirms the linear increase of $C_{\infty}$ with $\gamma_{t}$. The simulation results coincide with the analytical results. 


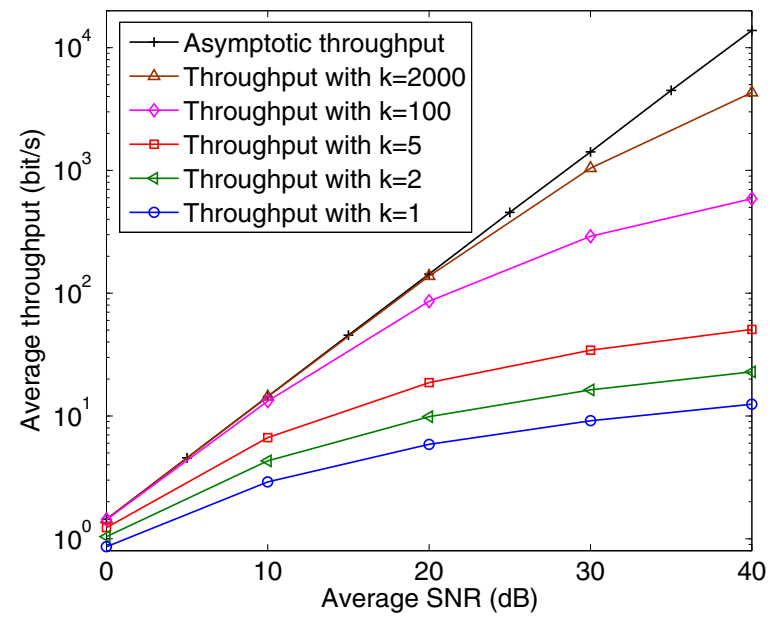

Fig. 4. Throughput comparison for different group sizes.

Fig. 4 illustrates how $C(k)$ gets closer to the asymptotic throughput $C_{\infty}$ with larger group size. In this simulation, the average throughput is plotted for different group sizes $k=1$, $2,5,100$, and 2000. It is shown that $C(k)$ indeed increases with $k$. When $k=2000, C(k)$ is very close to the asymptotic throughput for average SNR less than 30dB. Meanwhile, it is much higher than the unicast throughput, which corresponds to the curve with $k=1$. This implies that multicast is an effective way to obtain a throughput close to the infinite-bandwidth AWGN capacity with much limited bandwidth. This provides a strong incentive to apply multicast technique to broadband applications such as mobile TV in wireless OFDM systems.

The theoretic and simulation results of (19) are plotted in Fig. 5 for different values of $\epsilon$. It is observed that the difference between the theoretic and simulation curves are negligible for both $\epsilon=0.01$ and 0.001 . Moreover, the results show that the $\epsilon$-threshold group size indeed increases almost linearly with $\gamma_{t}$, as predicted in Theorem 4 .

\section{CONCLUSION}

In this paper, we investigate the optimal group strategy in multicast OFDM systems when the bandwidth resource allocated to a multicast group is proportional to the group size. Despite the decreasing data rate on each subcarrier with the increasing group size, our analysis shows that the expected throughput received by each user increases with the number of users in a group. When the group size goes to infinity, the average throughput approaches the capacity of the infinitebandwidth AWGN channel. Meanwhile, the group size that renders the throughput close to its asymptotic value grows almost linearly with the average transmit SNR. Our analysis matches the simulation results well.

\section{REFERENCES}

[1] S. Y. Hui and K. H. Yeung, "Challenges in the migration to $4 \mathrm{~g}$ mobile systems," IEEE Commun. Mag., vol. 41, pp. 54-56, December 2003.

[2] U. Varshney, "Multicast over wireless networks," Communications of the ACM, vol. 45, pp. 31-37, Dec. 2002.

[3] J. She, F. Hou, et al., "IPTV over WiMAX: key success factors, challenges, and solutions," IEEE Commun. Mag., vol. 45, pp. 87-93, Aug. 2007.

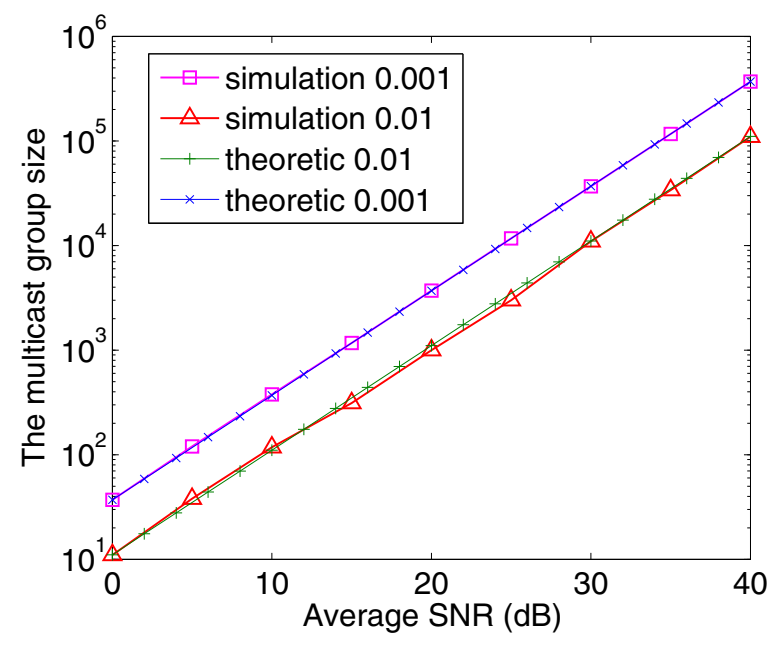

Fig. 5. The $\epsilon$-threshold multicast group size for $\epsilon=0.01$ and 0.001 .

[4] L. J. Cimini and N. R. Sollenberger, "OFDM with diversity and coding for advanced cellular internet services," in Proc. Globecom'1997. IEEE, Nov. 1997, pp. 305-309.

[5] T. M. Cover, "Broadcast channels," IEEE Trans. Inform. Theory, vol. IT-18, no. 1, pp. 2-14, Jan. 1972.

[6] L. Li and A. Goldsmith, "Capacity and optimal resource allocation for fading broadcast channels. i. ergodic capacity," IEEE Trans. Inform. Theory, vol. 47, no. 3, pp. 1083-1102, 2001.

[7] N. Jindal and A. Goldsmith, "Capacity and optimal power allocation for fading broadcast channels with minimum rates," IEEE Trans. Inform. Theory, vol. 49, pp. 2895 - 2909, Nov. 2003.

[8] M. Sharif, A. F. Dana, and B. Hassibi, "Differentiated rate scheduling for gaussian broadcast channels," in Proc. International Symp. Inform. Theory. IEEE, Sept. 2005, pp. 2179-2183.

[9] H. Won, H. Cai, et al., "Multicast scheduling in cellular data networks," in Proc. Infocom 2007. IEEE, May 2007, pp. 1172-1180.

[10] H. A. David, Order Statistics, 2nd ed. New York: Wiley, 1981.

[11] J. G. Proakis, Digital Communications, 3rd ed. New York: McGrawHill, 1995. 